\title{
The AL-SENSE Test Is Reliable for Detection of Second Trimester Amniotic Fluid
}

\author{
Marwan Odeh $^{1,2}$, Ella Ophir ${ }^{1,2}$, Hadar Kesari-Shoham ${ }^{3}$, Olga Maximovsky ${ }^{1}$, Jacob Bornstein ${ }^{1,2}$ \\ ${ }^{1}$ Department of Obstetrics and Gynecology, Western Galilee Hospital, Nahariya, Israel; ${ }^{2}$ Rappaport Faculty of Medicine, Technion, \\ Haifa, Israel; ${ }^{3}$ Commonsense, Caesarea, Israel. \\ Email: marwan20@bezeqint.net
}

Received December 13 ${ }^{\text {th }}, 2010$; revised April 10 $0^{\text {th }}, 2011$; accepted May $5^{\text {th }}, 2011$.

\begin{abstract}
Objective: To validate the reliability and stability of the AL-SENSE panty-liner for detection of amniotic fluid in the second trimester. Study design: Amniotic fluid drawn during amniocentesis between 16 to 23 weeks of gestation was used for the study. $100 \mu \mathrm{l}$ and $400 \mu \mathrm{l}$ of amniotic fluid, were dripped onto two panty liners, respectively. After 5 minutes the indicator strip was removed from the AL-SENSE, dried and examined. The color was recorded at time 0 and 5 minutes, and then every 10 minutes for the first 30 minutes, then at 1, 2, 6 and 12 hours. Results: 50 women were enrolled and 49 completed the study. 100\% of both volume samples changed color from yellow to blue during the first 5 minutes and remained stable after twelve hours of follow-up. In the $100 \mu \mathrm{l}$ subgroup, seven of the 49 samples (14.3\%) had a delayed color change and in the $400 \mu$ l subgroup one of $49(2.04 \%)$ had a delay in change of color. Conclusions: Amniotic fluid drawn during amniocentesis at weeks 16 - 23 of gestation have a positive, stable staining effect on the AL-SENSE panty liner. Hence, AL-SENSE may be reliable for detection of amniotic fluid leakage during the second trimester.
\end{abstract}

Keywords: Panty Liner, Amniocentesis, Second Trimester, Premature Rupture of Membranes

\section{Introduction}

Recently, a diagnostic panty-liner kit-“AL-SENSE” $($ (Commonsense, Caesarea, Israel) was developed to detect amniotic fluid leak. Its mechanism of action is described elsewhere [1]. The panty-liner has several advantages. Firstly, it is worn by the women for some hours, so it reflects a relatively lengthy time period and not a single point in time, which increases its sensitivity in detecting minor amniotic fluid leakage. In addition, it contains a unique polymer that is capable of differentiating amniotic fluid from urine.

In previous third trimester clinical studies [1,2], the AL-SENSE panty-liner test was documented as a sensitive tool for diagnosing premature rupture of the membranes (PROM) and differentiating amniotic fluid leaks from urinary incontinence. One study showed 100\% negative and $87.1 \%$ positive predictive values, with a sensitivity of $100 \%$ and specificity of $75 \%$. Another study showed $94.7 \%$ negative and $87 \%$ positive predictive value with a sensitivity of $96 \%$ and specificity of $84 \%$. The panty-liner was compared to a series of conventional tests such as speculum examination, nitrazine test, microscopic evaluation for ferning and ultrasound determination of intra-uterine amniotic volume [2]. These results were confirmed by another study [3] comparing the panty-liner test to speculum examination for detection of ruptured membranes; it found $98 \%$ sensitivity and $65 \%$ specificity.

Those studies enrolled women mostly at the third trimester. However, since amniotic fluid leakage during the second trimester has even more serious complications and poor outcomes than during the third trimester, the present study was undertaken to establish the AL-SENSE reliability in the second trimester and to validate the stability time of the positive color of AL-SENSE stains even after exposure to very small of early second trimester amniotic fluid. The amniotic fluid was drawn at genetic amniocentesis between 18 and 23 weeks of gestation.

\section{Materials and Methods}

The study was approved by the local Institutional Review Board (Helsinki committee) and written informed consent was obtained from each patient prior to enrollment. 
The AL-SENSE comprises a regular panty-liner and is worn as any other hygienic panty-liner. Inside the panty-liner the AL-SENSE contains one strip of (color) indicator that is sensitive to $\mathrm{pH}$ levels. The indicator strip reacts immediately to any contact with amniotic fluid or other vaginal discharge of $\mathrm{pH} 5.2$ or greater. Observing a change in the color of the $\mathrm{pH}$ indicator indicates either a leak of amniotic fluid or a urine leak. The indicators were placed in a drying box and observed to determine the stability of the stain.

During amniocentesis performed for medical reasons (primarily age over 35 years), between 18 and 23 weeks of gestation, $0.5 \mathrm{ml}$ of amniotic fluid, of the $30 \mathrm{ml} \mathrm{usu-}$ ally drawn, were used for this study.

Two volumes of amniotic fluid, $100 \mu \mathrm{l}$ and $400 \mu \mathrm{l}$, were dripped onto two AL-SENSE panty liners numbered 1 and 2, respectively. After five minutes the indicator strip was removed from the AL-SENSE and the strips were placed in the drying units.

The color status was recorded from both panty-liners immediately, after 5 and 10 minutes, and then every 10 minutes for the first 30 minutes and within 1, 2, 6 and 12 hours after removing the indicator strip and placing it in the drying unit.

\section{Statistical Analysis}

Statistical analyses were performed using $\mathrm{SAS}^{\circledR}$ v9.1 (SAS Institute, Cary N.C., USA). Descriptive statistics are presented in a table. Study results are presented as counts or percentages together with a $95 \%$ exact binomial confidence interval when relevant.

\section{Results}

Fifty (50) women were enrolled in the study; 49 (98\%) completed it. One patient was excluded due to a protocol deviation.

The distribution of gestational age is presented in Table 1; most women were in their $18^{\text {th }}-19^{\text {th }}$ week of gestation.

One hundred percent of samples in both volumes (95\% CI: $(92.75 \%$ - 100\%)) changed color from yellow to blue within five minutes and the color remained stable

Table 1. Distribution of gestational age by weeks.

\begin{tabular}{cll}
\hline Gestational Week & $\mathrm{N}$ & $\%$ \\
\hline 16 & 1 & 2.0 \\
17 & 3 & 6.1 \\
18 & 16 & 32.7 \\
19 & 21 & 42.9 \\
20 & 5 & 10.2 \\
21 & 1 & 2.0 \\
22 & 1 & 2.0 \\
23 & 1 & 2.0 \\
Total & 49 & 100.0 \\
\hline
\end{tabular}

throughout the twelve hour follow-up period. Initial indicator color change did not occur at time 0 for all the samples. In the $100 \mu \mathrm{l}$ subgroup 7 of the 49 samples, or $14.3 \%$ (95\% CI: (5.94\% - 27.24\%)), had a delayed color change and in the $400 \mu$ l subgroup 1 of 49 , i.e. $2.04 \%$ (95\% CI: $(0.05 \%-10.85 \%))$, showed a delay in color change.

\section{Discussion}

In the present study the AL-Sense panty-liner was found to be very sensitive and changed its color in response to a very small amount of second trimester amniotic fluid obtained at amniocentesis. The color change was stable for 12 hours, the time determined in advance as the completion of the study. So far, the validity of the AL-Sense panty liner has been proven in clinical studies in diagnosing premature rupture of membranes in the third trimester [1-3]. However, the effectiveness of this test had not been tested in second trimester pregnancies.

This study utilized amniotic fluid obtained during amniocentesis. Therefore, the effectiveness of this test should be verified in a clinical setting, as the color of the panty-liner may change in reaction to vaginal secretions when infections that cause elevated $\mathrm{pH}$ are present (as stated by the manufacturer). Nevertheless, Mulhair et al. [3] found that women with positive high vaginal swabs did not differ significantly and nine women with positive high vaginal swab had a negative test (no color change).

The high $\mathrm{pH}$ level of amniotic fluid (7.42) even at very early stages of pregnancy [4], the ability of the panty-liner to detect it, even in very small amounts (100 $\mu \mathrm{l})$, and the long term stability of the stain makes it a very useful tool in diagnosing cases with premature rupture of the membranes in the second trimester. This period of pregnancy may be associated with significant management dilemmas due to the high percentage of fetal, neonatal and maternal complications with PROM at that time [5-7]. The high sensitivity of this test in clinical studies $(100 \%)$ makes it very helpful in reassuring patients that membranes have not ruptured.

A drawback of this test is its low specificity; hence, when the test is positive the diagnosis should be verified by other means. However, in this study, as we dripped amniotic fluid directly on the panty-liner and there was no contact with vaginal discharge or other fluide, we did not include a positive/negative control.

Another possible use of this test is in patients experiencing post-amniocentesis fluid leakage. Since most of these cases may be followed expectantly $[8,9]$ the test can be used to indicate cessation of amniotic fluid leakage (when a positive test become negative).

In conclusion, the panty-liner is effective in the second trimester of pregnancy and can be used at that time 
to detect small amounts of amniotic fluid leakage, and to exclude its presence if the leakage consists of urine. It is very sensitive and detects small amounts of amniotic fluid drawn during amniocentesis.

As the present study has been conducted in vitro, the efficacy of AL-Sense should be further verified in clinical studies of women with fluid leakage during the second trimester, in the same way as it has been tested in women during the third trimester.

\section{Conflict of Interests}

Hadar Kesari-Shoham is employed by the manufacturer of the AL-SENSE panty-liner.

\section{REFERENCES}

[1] J. Bornstein, A. Geva, I. Solt, V. Fait, A. Schoenfeld, H. K. Shoham and J. Sobel, "Nonintrusive Diagnosis of Premature Ruptured Amniotic Membranes Using a Novel Polymer," American Journal of Perinatology, Vol. 23, No. 6, 2006, pp. 351-354. doi:10.1055/s-2006-947159

[2] J. Bornstein, G. ohel, Y. Sorokin, K. Z. Reape, O. Shmaider, H. Kessary-Shoham and E. Ophir, "Effectiveness of a Novel Home-Based Testing Device for the Detection of Rupture of Membranes," American Journal of Perinatology, Vol. 26, No. 1, 2009, pp. 45-50. doi:10.1055/s-0028-1095183

[3] L. Mulhair, J. Carter, L. Poston, P. Seed and A. Briley, "Prospective Cohort Study Investigating the Reliability of the AmnioSense ${ }^{\mathrm{TM}}$ Method for Detection of Spontaneous Rupture of Membranes," An International Journal of $\mathrm{Ob}$ stetrics and Gynaecology, Vol. 116, No. 2, 2009, pp. 313-

\section{8. doi:10.1111/j.1471-0528.2008.01828.x}

[4] E. Jauniaux, D. Jurkovic, B. Gulbis, W. P. Collins, J. Zaidi and S. Campbell, "Investigation of Acid-Base Balance of Coelomic and Amniotic Fluids in Early Human Pregnancy," American Journal of Obstetrics and Gynecology, Vol. 170, No. 5, 1994, pp. 1365-1369.

[5] A. Greenough and M. Blott, "Neonatal Outcome after Prolonged Rupture of the Membranes Starting in the Second Trimester," Archives of Disease in Childhood, Vol. 63, No. 10, 1988, pp. 1146-1150. doi:10.1136/adc.63.10_Spec_No.1146

[6] A. Farooqi, P. A. Holmgren, S. Engberg and F. Serenius, "Survival and 2-Year Outcome with Expectant Management of Second-Trimester Rupture of Membranes," $\mathrm{Ob}$ stetrics and Gynecology, Vol. 92, No. 6, 1998, pp. 895901. doi:10.1016/S0029-7844(98)00287-7

[7] J. U. Hibbard, M. C. Hibbard, M. Ismail and E. Arendt, "Pregnancy Outcome after Expectant Management of Premature Rupture of the Membranes in the Second Trimester," The Journal of Reproductive Medicine, Vol. 38, No. 12, 1993, pp. 945-951.

[8] R. B. Gold, G. L. Goyert, D. B. Schwartz, M. I. Evans and L. A. Seabolt, "Conservative Management of Second Trimester Post Amniocentesis Fluid Leakage," Obstetrics and Gynecology, Vol. 74, No. 5, 1989, pp. 745-747.

[9] A. F. Borgida, A. A. Mills, D. M. Feldman, J. F. Rodis and J. F. X. Egan, "Outcome of Pregnancies Complicated by Ruptured Membranes after Genetic Amniocentesis," American Journal of Obstetrics and Gynecology, Vol. 183, No. 4, 2000, pp. 937-939. doi:10.1067/mob.2000.108872 